\title{
м. Goebeler Psoriasis - Krankheit und Therapie im Wandel?!
}

\section{Psoriasis - Disease and Treatment in Flux?!}

Die Dermatologie ist ein ziemlich konservatives Fach: Wir setzen noch heute Therapien ein, die sich mitunter, wie z.B. Teerpräparate, bis in die Antike zurückführen lassen. Auch eines der bis heute wichtigsten Dermatika für die Behandlung der Psoriasis, Cignolin, findet seit mittlerweile 90 Jahren in der klinischen Praxis Anwendung. Es wurde 1916 durch Galewski und Unna eingeführt, als kriegsbedingt die Versorgung mit dem aus Brasilien importierten Goa-Pulver, das aus dem Ararobabaum gewonnen wurde und zur Herstellung des vormals eingesetzten Chrysarobins notwendig war, stockte. Das Konzept der Behandlung schwerer Psoriasisformen mit Folsäureantagonisten, erstmals 1951 von Gubner vorgestellt, ist mittlerweile ebenfalls in die Jahre gekommen. Das zunächst verwendete Aminopterin wurde später durch Methotrexat (MTX) ersetzt, welches bis heute einen der Goldstandards nicht nur in der Behandlung der Psoriasisarthritis darstellt. Weitere Meilensteine in der Psoriasistherapie waren die Einführung des Ciclosporin in den 1970er Jahren, des Vitamin D und seiner Analoga, deren Ursprünge bereits in die 30er Jahre des vergangenen Jahrhunderts zurückreichen, der Retinoide (1970er Jahre), der Fumarsäure (1959) und schließlich der modernen Phototherapieverfahren.

Nach Jahren relativen Stillstandes in der Entwicklung und Einführung innovativer Antipsoriatika ist jetzt eine Zeit angebrochen, in der völlig neue, sich als „,rational“ verstehende Therapieansätze in die klinische Dermatologie eingeführt wurden bzw. werden. Wenngleich die Ursachen der Psoriasis weiterhin im Dunkeln liegen - über genetische Defekte und putative Autoantigene besteht bis heute nur wenig Klarheit -, so kann mittels neuer Substanzklassen immerhin auf der Effektorebene mehr oder weniger spezifisch mit dem Krankheitsprozess interferiert werden. Diese innovativen Substanzen umfassen nicht allein biologicals, biotechnologisch hergestellte, meist großmolekulare Proteine, in der Regel Fusionsproteine und Antikörper, sondern auch niedermolekulare Substanzen, die in ähnlich spezifischer Weise in pathophysiologische Prozesse eingreifen. Zahlreiche solcher Substanzen werden derzeit in klinischen Studien evaluiert.

Parallel zu diesen Entwicklungen ergibt sich der Eindruck, dass das Krankheitsbild der Psoriasis ärztlicherseits wie auch in Wissenschaftskreisen in den letzten Jahren eine neue Bewertung zu erfahren scheint. Die Erkenntnis, dass der Psoriasis (auch) eine T-Zell-vermittelte Autoimmunpathogenese zugrunde liegt, hat sie gewissermaßen in den Kreis der „richtigen“ (Auto)Immunerkrankungen aufrücken lassen. Sichtbar wird dies beispielsweise an dem wachsenden wissenschaftlichen Interesse der Rheumatologen an der Psoriasisarthritis, nachdem diese zuvor eher ein Nischendasein führte. Auch die Erkenntnis, dass schwere Formen der Psoriasis mit einer erhöhten kardiovaskulären Morbidität und Mortalität einherzugehen scheinen, unterstützt die veränderte Wahrnehmung der Psoriasis als ernste Erkrankung. Inwieweit sich im Hinblick auf die Psoriasis Erkenntnisfortschritt und Wahrnehmungswandel in der ärztlich-wissenschaftlichen community und die Interessenslage der pharmazeutisch-biotechnologischen Industrie gegenseitig bedingen, wäre wohl einer weiteren Analyse wert... 
Im Mittelpunkt unserer ärztlichen Bemühungen steht selbstverständlich der von der Psoriasis betroffene Patient - er hat sicherlich schon immer seine Erkrankung als ernst und beeinträchtigend wahrgenommen. Neuere Studien untermauern dies: So wird der Einfluss einer Psoriasis auf die Lebensqualität als vergleichbar ausgeprägt empfunden wie der von anderen chronischen und zum Teil lebensbedrohlichen Krankheiten wie z.B. Krebserkrankungen und Herzinfarkten (siehe auch Beitrag von Schmid-Ott und Schallmayer in dieser Ausgabe der Aktuellen Dermatologie). In diesem Spannungsfeld ist es die Aufgabe von uns Dermatologen, aus der (glücklicherweise) größer werdenden Anzahl therapeutischer Optionen gemeinsam mit dem Patienten die im individuellen Fall beste Therapie auszuwählen. Dass (unglücklicherweise) zunehmend wirtschaftliche Zwänge mit diesem Findungsprozess interferieren, muss hier nicht näher ausgeführt werden. Schließlich ist es auch unsere Aufgabe, KoMorbiditäten der Psoriasis frühzeitig zu erkennen und adäquat zu berücksichtigen. Nicht zuletzt sollten wir dazu beitragen, das Krankheitsverständnis der Betroffenen, beispielsweise durch Schulungsangebote, zu unterstützen und die Kooperation mit
Selbsthilfegruppen zu suchen. $\mathrm{Zu}$ einer optimierten Betreuung von Psoriasispatienten können Strukturen beitragen, wie sie mit dem Kompetenzzentrum Psoriasis an der Klinik für Dermatologie, Venerologie und Allergologie des Universitätsklinikums Mannheim gegenwärtig implementiert werden.

In dem vorliegenden Themenheft der Aktuellen Dermatologie werden unterschiedliche Facetten der Psoriasis beleuchtet. Einzelne Beiträge sind der Pathophysiologie, der Psoriasisarthritis und den psychosozialen Aspekten der Psoriasis gewidmet. Der Schwerpunkt dieser Ausgabe liegt allerdings auf den vielfältigen therapeutischen Ansätzen, die in durchaus kontroverser Weise vorgestellt werden. Wohl allen Autoren ist bei der Vorbereitung dieses Hefts wieder einmal bewusst geworden, wie dringend notwendig über Zulassungsstudien hinausgehende, sorgfältig konzipierte und mit hoher Qualität durchgeführte Studien zur Psoriasis sind, die unabhängig von Partikularinteressen unterschiedliche Therapiekonzepte zum Wohle der Patienten miteinander vergleichen. 